\title{
Research on the Echo Chamber Effect
}

\author{
Jiuyu Chen ${ }^{1 *}$ \\ ${ }^{I}$ Social Science, New York University Shanghai, Shanghai, 200135, China \\ *Corresponding author. Email: jc10400@nyu.edu
}

\begin{abstract}
This essay explores the concept of the echo chamber effect and the validity of this concept. In this essay, how the echo chamber effect emerges as a product of the Internet is reviewed. This essay also compares the differences and similarities between two similar concepts, namely the echo chamber effect and the filter bubble. Many research papers investigate the negative impacts of the echo chamber effect. However, whether the echo chamber effect is as intimidating as these researches suggest is worth arguing. Though the echo chamber effect exists within single platforms, a high variety of media diets offsets the negative consequences of the echo chamber effect.
\end{abstract}

Keywords: Echo Chamber, Filter bubble, Internet, Media diversity

\section{INTRODUCTION}

The invention of the Internet has brought many advantages to our daily lives, however, it could also change how we think and behave in an unexpected way. When you're browsing through the Internet, have you noticed that when searching for the same things, your results could be significantly different from your friends' results. This is because you might have fallen into the pitfall of the echo chamber effect. The echo chamber effect is a metaphorical way of describing an Internet environment and mechanism where only partial information or information from only one side of the fact was shared [1][2]. The term echo chamber effect was a product of the Internet and individualized search engines, so research on the echo chamber effect has been a relatively new topic. With that being said, there are still tons of research available in the field of academic discussion to be reviewed. However, most of these research shares similar limitations in that they usually focus on single media or platforms. It is financially and technically demanding to conduct large-scale research on the Internet. Thus, there has been a dispute about whether the echo chamber effect is valid. In this research, the history of the echo chamber effect, the similarities and differences between the echo chamber effect and the negative impacts of the echo chamber effect will be explored. After that, the validity of the echo chamber effect will be discussed. This research sheds light on the general information of the echo chamber effect. It also views the creation of this term with a critical lens.

\section{INTERNET AND THE ECHO CHAMBER EFFECT}

The rapid expansion of the Internet leads to new waves of exposure to information and new ways of consuming information. Some hold positive views towards this change, believing that the Internet enables a more diverse form of public debate on various agendas. However, theories like the echo chamber effect suggest otherwise.

The echo chamber is a term most commonly used in digital media research and sometimes used in political research. In a media context, it refers to a situation where beliefs are amplified and reinforced by communication and repetition inside a closed system and insulated from rebuttal. This usually occurs when people find likeminded people online. They hold similar views on certain topics. When they voice their opinions, they always receive positive and similar feedback, though it could sometimes be distorted and deviate from the actual fact. The positive feedbacks reinforce their existing beliefs until at some point, most people in this closed system believe the spread truth and hold it as an extreme, absolute truth. Because of their continuous selfconfirmation of certain beliefs, they've formed a strong belief system existing within themselves which makes it hard for anyone in this system to accept rebuttal or contrasting views [3]. It occurs as similar opinions are spread within a small, closed discourse system. These voices are repeated and paraphrased or even exaggerated until most people in the room assume the knowledge is 
true. The repeated process of hearing echoes of their own voices reinforced their existing belief system. By forming a strong closure of a believe system, the echo chamber shuts out rebuttal and possible contrasting voices. Thus, the echo chamber has a negative effect in encouraging diverse conversations and reducing bias.

The echo chamber effect is a result of shifted media environment. The media has undergone a major shift in its consumption channels. Especially in recent years, there is an increasing tendency for the consumption of information to shift from traditional media towards media driven by the Internet like social media and search engines. This can be seen evidently from the shifting access of the consumption of news. According to the Reuters Institute Digital News Report, which was conducted in 26 countries with over 50000 participants, 23 percent of respondents primarily use digital channels to obtain news, while 44 percent regard digital and traditional media as equally important In these 26 countries, digital media and online media are equally important news sources. When consuming online news, social media and search engines are people's top 2 choices, with roughly 40 and 33 percent of the respondents selecting them respectively, followed by news aggregators with 12.5 percent of the respondents [4]. Compared with the news environment from a century ago where newspapers and television were still the mainstream media of the society, there has been a major shift of methods in media consumption.

\section{THE ECHO CHAMBER EFFECT AND FILTER BUBBLE}

The echo chamber effect and the filter bubble are both metaphoric descriptions of a media phenomenon in which only partial information is shared. In today's scholarly discussions, the two related concepts filter bubble and echo chamber are used more and more interchangeably since these two concepts are both mainly used in the media sphere. But it could also result from the fact that both terms weren't well defined by their creators. The term echo chamber effect first appeared in Cass Sustein's 2001 book Echo Chambers: Bush v. Gore, Impeachment, and Beyond [5]. The term filter bubble is coined much later in 2011 by Eil Pariser in his book Filter Bubble: What the Internet Is Hiding from You[6]. It is worth noting that both Sustein and Pariser weren't really specialists in media and communication studies. They are a legal scholar and internet activist who discovered alarming anecdotes in their field of specialty and further expand them into a hypothesis. Thus, whether these hypotheses are reliable and valid requires further experimental testing.

The two terms, echo chamber and filter bubble, themselves have distinctive conceptual differences. However, due to the blurriness of definition, they are sometimes misused by media and even scholars. The echo chamber effect is a belief system where only homogeneous information is accepted while heterogeneous information is either ignored or discredited. The voices entering the echo chamber are actively excluded. There is a structural manipulation of trust existing inside the echo chamber by discrediting all outside sources [7]. This is why echo chambers are particularly strong. They are embedded in a human tendency to seek approval and reject different voices. Also, the echo chamber brings with itself a closed process of reinforcement which allows it to strengthen itself repeatedly.

Compared to the echo chamber effect, the filter bubble is weaker in its mechanism and effect. The filter bubble is based on search engines and personalized functions. It creates a "personalized universe of information" by looking at people's search histories and using algorithms to calculate their preferences. It only creates a lack of information that results from a systematic defect of the system which can be fixed by partially changing how search engines work and providing relevant information from a different angel. However, the echo chamber is formed more on the cognitive level than a systematic level. To alter echo chamber member's thoughts and behaviors, it is not enough to simply provide them with opposite views, because the echo chamber has caused them to create a strong belief system that cannot be easily altered.

\section{THE ECHO CHAMBER EFFECT AND NEGATIVE EFFECTS}

There are many disadvantages to the echo chamber. As defined, the echo chamber effect refers to a situation where beliefs are amplified and reinforced by communication and repetition inside a closed system and are insulated from rebuttal. In other words, the echo chamber effect creates "a single story" [8]. In Chimamanda Ngozi Adichie's speech "The danger of a single story", she puts forward the idea of "a single story", which means to look at a person, a region or a thing from one perspective and one perspective only. She argued that in this way, not only can one fail to recognize the whole picture of a thing, their one-sided judgement could also dehumanize people into a single word or subjective opinion. People's dignity is broken in unintended microaggressions of people standing on moral high ground [8]. Similarly, people inside the echo chamber can fall into the trap of "a single story". When they use their partial knowledge to judge something, they may very likely end up with an incomplete judgement.

On top of this, the mass aggregation of echo chambers could also pose threats to different types of democracy. Liberal democrats emphasize the importance of free conscious and personal freedom of choice. The echo chamber undermines liberal democracy by dismissing outside voices and opinions which potentially 
limits their awareness and freedom of choice. Deliberative democrats try to increase the quality of information, from which they discover truth and disagreements. Under the context of echo chamber, since opposite voices are deliberately excluded from the group, disagreements are easily identified between different echo chambers while truth can hardly be acknowledged by any group. The echo chamber also diminishes mutual respect and understanding. At the same time, it reduces human's ability of to accept different ideas and establish better arguments on opposing views. Contestatory democracy on the other hand relies on effective channels for contests. One major factor of the channels is the ability to have key information on important issues so that citizens are able to make informed decisions. Media usually carries the burden of providing key information to the public. However, the echo chamber could exclude these valid channels, thus resulting in similar missing information as filter bubble[9].

The echo chamber effect is certainly frightening if it is true. While some empirical studies support these concerns, there are also empirical studies that find no evidence for this phenomenon. This research question is whether the echo chamber has an effect on people's thoughts and beliefs? The hypothesis is that the echo chamber has an effect on people's thoughts and beliefs.

\section{DISCUSSION AND MEDIA DIVERSITY}

Inspecting on various researches, there are many supporting the hypothesis of the echo chamber and many that do not. However, it seems that those researches which have found evidence of the echo chamber effect are all conducted on the premise of single media. For example, a study by Bakshy et al discovered that news with selective exposure enjoys significantly higher attention. "People encountered roughly $15 \%$ less crosscutting content in news feeds due to algorithmic ranking and clicked through to $70 \%$ less of this cross-cutting content." [10]. In other words, with the influence of the echo chamber effect, people are more likely to encounter narrative from a one-sided perspective and they are much more likely to view contents that provide only one side of the story than those that are more objective. Though Bakshy's study was reliable and valid, one important premise that cannot be ignored is that he only conducts the study on Facebook, which means this study is under the assumption that these people receive information from Facebook only. However, another study reveals that most people live in a "high-choice media environment", which means that most people actually consume their information from more than one media source[11]. The polarization of political or other opinions may seem obvious on one social media platform, but when it comes to an overview of the entire media environment, no significant echo chamber exists. People always encounter things that they disagree with. To confirm the information, they usually inspect various sources from different media. It is within this process that they are slowly revising their own point of view. The moment that they are exposed to different voices, they are hearing more than just the echoes of their own opinions. Though extreme echo chamber might end up with a strong excluding effect for outside voices, they allow contrasting views to exist before the echo chambers are formally formed.Even though it can be observed in a single media or platform that like-minded people usually gather together and produce homogeneous opinions that function as echoes, it is difficult to study how different types of media influence people, how heterogeneous content across different media can unexpectedly be presented to the audience without their conscious awareness. In other words, if people are exposed to a "high-choice media environment", they are less likely to fall into echo chamber. According to the study, $92 \%$ of the respondents live in a media environment with high diversity, with only $8 \%$ of the respondents live in relatively singular media environment[11]. Despite being more susceptible to the echo chamber, $8 \%$ of respondents stated that they have little interest in politics. In my literature review, the most destructive effect of the echo chamber lies in its disruptive effects towards different types of democratic regimes, since the echo chamber adds the possibility to underinformed decisions and political biases. However, since the population that is most easily influenced by echo chamber are not interested in politics, the echo chamber effect on politics should be less than how much people concerned.

Conover et al. has found similar results in his study on Twitter users. They found that it seems that the information and networks on Twitter are polarized[12]. Thus, when we only look at Twitter, it is possible to spot echo chambers with polarized voices. However, as Dubois points out, political information can be attained from various channels. The Twitter research doesn't account for news channel $\mathrm{CNN}$ or $\mathrm{BBC}$ news from political websites, television channels or newspaper like Washington Post, where information consumers can encounter a broader spectrum of political ideas.

\section{CONCLUSION}

The echo chamber has a destructive effect on various democratic regimes. It is also harmful to mature public discourses. However, there are also many opposing voices asserting that the echo chamber effect has little impact on our daily lives and thoughts.

This research explores the history, the characteristics and negative effects of the echo chamber effect. Several research papers have found supporting evidence on the echo chamber effect are all conducted on single media such as Facebook or Twitter, but as other research papers point out, the majority of people in our society have more diverse media diets, allowing them to consume opinions 
with variety and reduce their chances of falling into the echo chamber. To conclude, though echo chamber effect exists on single media platforms, it creates less effect than it is advertised because of the high-choice media environment.

\section{ACKNOWLEDGMENT}

I would like to express my deep gratitude to Professor Edward Schiappa for his inspiring lectures and useful advice. I would also like to thank Ms. Huang and Ms. Song, for her advice and assistance in keeping my progress on schedule.

Finally, I wish to thank my parents for their support and encouragement throughout my study.

\section{REFERENCES}

[1] Jamieson, Kathleen \& Cappella, Joseph. (2008). Echo Chamber: Rush Limbaugh and the Conservative Media Establishment.

[2] Sunstein, C. (2002). The Law of group polarization. Journal of Political Philosophy, 10(2), 175-195. doi: 10.1111/1467-9760.00148

[3] Parry, Robert (2006-12-28). "The GOP's \$3 Bn Propaganda Organ". The Baltimore Chronicle.

[4] Newman, Nic, Richard Fletcher, David A. L. Levy, and Rasmus Kleis Nielsen. 2016. Digital News Report 2016. Oxford.

[5] Sunstein, C. R. (2001). Echo Chambers: Bush v. Gore, Impeachment, and Beyond. Princeton: Princeton University Press.

[6] Pariser, E. (2011a). The Filter Bubble: What the Internet Is Hiding from You. London: Penguin.

[7] ""Echo chambers," polarization, and the increasing tension between the (social) reality of expertise and the (cultural) suspicion of authority". uva.theopenscholar.com.

[8] Adichie, Chimamanda Ngozi. "The Danger of a Single Story.” TED Talks, www.ted.com/talks/chimamanda_ngozi_adichie_th e_danger_of_a_single_story/transcript.

[9] Bozdag, E., van den Hoven, J. Breaking the filter bubble: democracy and design. Ethics Inf Technol 17, 249-265 (2015). https://doi.org/10.1007/s10676-015-9380-y

[10] Bakshy, Eytan; Messing, Solomon; Adamic, Lada A. (2015-06-05). "Exposure to ideologically diverse news and opinion on Facebook". Science. DOI:10.1126/science.aaa1160.

[11] 13. Elizabeth Dubois \& Grant Blank (2018). The echo chamber is overstated: the moderating effect of political interest and diverse media, Information, Communication \& Society, 21:5, 729-745, DOI: 10.1080/1369118X.2018.1428656

[12] Bakshy, Eytan; Messing, Solomon; Adamic, Lada A. (2015-06-05). "Exposure to ideologically diverse news and opinion on Facebook". Science. DOI:10.1126/science.aaa1160. 